\title{
RELAÇÃO ENTRE O ENFRENTAMENTO RELIGIOSO/ESPIRITUAL COM A CAPACIDADE FUNCIONAL DE HEMIPARÉTICOS SUBMETIDOS A FISIOTERAPIA EM CIRCUITO DE TREINAMENTO
}

Andressa Sampaio Pereira, Caroline Nunes Gonzaga, Silas de Oliveira Damasceno, Katiane Mayara Guerrero, Alice Haniuda Moliterno, Isabela Bortolim Frasson, Isabella Menezes Silva, Guilherme Yassuyuki Tacao, Lúcia Martins Barbatto, Augusto Cesinando de Carvalho

Universidade Estadual Paulista “Júlio Mesquita Filho" - UNESP, Departamento de Fisioterapia, Curso de Fisioterapia, Presidente Prudente, SP. E-mail: andressa fisio2013@hotmail.com.

\section{RESUMO}

A área de pesquisa entre a espiritualidade e a doença está cada vez mais em evidência, todavia, não foi encontrada na literatura a relação entre a espiritualidade/religiosidade com indivíduos pós-AVE. Correlacionar o Coping Religioso Espiritual com a capacidade funcional dos hemiparéticos submetidos a Fisioterapia em Grupo no formato de Circuito (FGCT). Estudo clínico transversal com 8 hemiparéticos crônicas em FGCT. Foi aplicado a avaliação inicial e após 8 semanas de FGCT a avaliação final com a aplicação da escala CRE-Breve e da Timed Up and Go (TUG). Não houve correlação entre o questionário CRE-breve com o TUG $(r=-0,618$ e $p=0,102)$, mas o effect size mostrou um grande efeito clínico $(0,94)$. A FGCT não promoveu melhora na mobilidade funcional ao final de 8 semanas e nem correlação significante entre CRE e TUG, todavia, foi evidenciado efeito positivo ao enfrentamento da doença e um grande efeito clínico entre as avaliações aplicadas.

Palavras-chave: acidente vascular cerebral, paresia, exercícios em circuito, espiritualidade, coping

\section{RELATIONSHIP BETWEEN RELIGIOUS/SPIRITUAL COPING AND FUNCTIONAL CAPACITY OF HEMIPARETICS SUBMITTED TO PHYSIOTHERAPY IN TRAINING CIRCUIT FORMAT}

\begin{abstract}
The research field between spirituality and disease is increasingly evident, however, the relationship between spirituality/religiosity coping in post-stroke individuals has not been found in the literature. To correlate spiritual/religious coping and functional capacity in hemiparetics submitted to Group Physiotherapy in Circuit Format (GPCF). Cross-sectional study with 8 chronic hemiparetic patients in GPCF. At first, the initial evaluation was applied and after 8-weeks intervention, following CRE-brief and Timed Up and Go (TUG). There was no correlation between the CRE-brief questionnaire with TUG $(r=-0.618$ and $p=0.102)$, but the effect size showed a large clinical effect (0.94). The GPCF did not promote improvement in functional capacity at the end of 8-weeks intervention and no significant correlation between CRE-brief and TUG. However, it was demonstrated positive effect in fighting of the disease and a great clinical effect among the applied evaluations.
\end{abstract}

Keywords: Stroke, paresis, circuit exercises, spirituality, coping. 


\section{INTRODUÇÃO}

Segundo dados do Instituto Brasileiro de Geografia e Estatística (41) somente $8,0 \%$ da população brasileira não têm religião e para a Organização Mundial de Saúde (OMS), saúde é definida como "um estado de completo bem-estar físico, mental e social", sendo frequentemente visto como um componente inseparável do bem-estar espiritual ${ }^{1,2}$.

A área de pesquisa entre a espiritualidade e a doença está cada vez mais em evidência ${ }^{3}$. A orientação espiritual contribui significativamente para a saúde mental, pois há estudos que apontam que há uma relação entre a religiosidade e a espiritualidade como indicadores positivos e/ou negativos na saúde ${ }^{4,5,6}$.

Assim sendo, a espiritualidade deriva do termo spiritus que significa sopro de vida e está relacionado a crenças e valores, que podem estar intimamente envolvidas com a capacidade de suportar sentimentos diversos, podendo ou não incluir a participação de uma religião; em contrapartida, a religiosidade está relacionada a prática de tradições sagradas. Estudos apontam que a espiritualidade em hospitais tem sido associada a uma redução no risco de depressão e suicídio, e tem se tornado uma estratégia para facilitar comportamentos saudáveis e de maior otimismo $^{7,8,9}$.

É visto que a capacidade de lidar com as circunstâncias oriundas da vida através do enfrentamento religioso e espiritual (CRE) tem sido crescente, no entanto, a literatura nos mostra que é possível ter um CRE positivo (CREp) ou um CRE negativo (CREn). O CREp engloba comportamentos e práticas que levam a um efeito benéfico do indivíduo, através do amor e da proteção de Deus ou com forças transcendentais, mas o CREn envolve atitudes e comportamentos relacionados com o sofrimento e repercussões prejudiciais desencadeadas pela doença, sendo uma forma de punição divina ${ }^{10,4}$.

Os indivíduos com doenças crônicas como o câncer, AVE, doença de parkinson, entre outros, necessitam de uma atenção individualizada, uma vez que esse momento pode apresentar significações diferenciadas para cada pessoa. Desta maneira, o cuidado na espiritualidade passa a ser fundamental.

Lesões no sistema corticoespinhal decorrente do AVE, resultam em danos sensóriomotores, e tem como sequela mais evidente a hemiparesia, que tem como consequência a espasticidade, deficiência motora e fraqueza muscular no hemicorpo contralateral à lesão ${ }^{11}$, é um problema de saúde mundial e uma das principais causas de incapacidade.

Essas alterações, podem levar a restrições de participação social favorecendo uma maior dependência durante a realização das atividades da vida diária ${ }^{12}$. Como resultado disso, esses indivíduos ficam mais suscetíveis ao sedentarismo, o que pode resultar em prejuízos motores, apontando a necessidade de participação em programas que envolvam exercícios físicos funcionais $^{13,14}$.

Mesmo com a reabilitação, cerca de um quinto dessa população sofre um declínio significativo na mobilidade, e menos de $50 \%$ dos pacientes conseguem caminhar independentemente na comunidade novamente. Um dos métodos terapêuticos utilizados é a fisioterapia em grupo no formato de circuito de treinamento (FGCT), que é uma forma terapêutica viável para as pessoas que apresentam dificuldades durante a execução das atividades de vida diária $^{15,16}$.

O método é composto por um conjunto de atividades específicas, onde o indivíduo é sempre desafiado na sua capacidade máxima. Isto permite que a FGCT seja um modelo terapêutico que não precise necessariamente ser uma adição à terapia habitual ${ }^{17}$. Entretanto, não foi encontrado na literatura a relação entre a espiritualidade/religiosidade com indivíduos pós $\mathrm{AVE}$, tendo em vista isso, o objetivo é correlacionar o CRE com a capacidade funcional dos hemiparéticos submetidos a FGCT. 


\section{MATERIAIS E MÉTODOS}

Para realização deste estudo clínico transversal foram recrutados hemiparéticos em atendimento de FGCT no Centro de Atendimento de Fisioterapia e Reabilitação (CEAFIR) da Universidade Estadual Paulista "Júlio de Mesquita Filho" (UNESP), Faculdade de Ciências e Tecnologia (FCT), campus de Presidente Prudente.

Foram incluídos no estudo, indivíduos com encaminhamento médico e hemiparesia com tempo de lesão $\geq$ há 6 meses, capazes de realizar marcha com ou sem auxílio de órtese, que apresentassem fraqueza e/ou alteração de tônus, identificadas pelos escores diferentes de zero na escala modificada de Ashworth $^{18}$; além de ausência de déficits cognitivos avaliados pelo MiniExame do Estado Mental (ponto de corte para indivíduos analfabetos 18/19 e para indivíduos com instrução escolar $24 / 25)^{19}$.

Todos os voluntários foram informados sobre os objetivos e procedimentos do estudo e após concordarem, assinaram um termo de consentimento livre e esclarecido submetido ao Comitê de Ética da FCT - UNESP (CAAE:81658317.0.0000.5402).

Antes dos participantes do estudo serem submetidos a FGCT, foi aplicado a avaliação inicial (AV1) e após 8 semanas de intervenção, foi realizada a avaliação final (AV2). As escalas utilizadas foram:

- CRE-Breve (CREb): Essa escala foi traduzida, validade e adaptada para a cultura brasileira. Possui 49 itens e seus objetivos estão relacionados ao grau de enfretamento da doença e seus desdobramentos na busca de conforto espiritual, intimidade com Deus, transformação de vida, e a busca do bem-estar físico, psicológico e emocional. Os itens estão relacionados aos fatores de Coping Religioso/Espiritual Positivo (CREp) somando 34 itens, e fatores de Coping Religioso Espiritual Negativo (CREn) somando 15 itens. É calculado o total (CREt) de estratégias pela pessoa para o enfrentamento de estímulos estressores, obtidos pela média entre o índice CREp e a média da inversão das respostas aos itens do CREn. São mantidos valores entre 1,0 e 5,0, e os parâmetros utilizados para a análise das médias do CRE podem ser: nenhuma ou irrisória (1,0 a 1,5); baixa $(1,51$ a 2,5); média $(2,51$ a 3,5); alta (3,51 a 4,5); altíssima $(4,51$ s 5,0), e quanto maior seu valor, maior é a utilização do CREp. As respostas foram dadas em escala do tipo Likert de cinco pontos, que variam de 1- nem um pouco; 2- um pouco; 3- mais ou menos; 4- bastante e 5 muitíssimo ${ }^{20}$.

- Timed Up and Go: é um teste que avalia a mobilidade funcional e a marcha. Para a realização do teste foi colocado o indivíduo sentado numa cadeira confortável, com apoio para as costas e braços, utilizando seus calçados usuais e seu dispositivo de auxílio à marcha, e após o comando "vá", o indivíduo devia se levantar da cadeira e andar um percurso linear de 3 metros, com passos seguros, retornar em direção à cadeira e sentar-se novamente. $O$ tempo requerido para completar a tarefa foi mensurado em segundos por um cronômetro ${ }^{21}$.

A FGCT ocorreu em um espaço com 14 tipos de exercícios diferentes, onde cada indivíduo ficou por um período de 3 minutos em cada estação; além disso, os atendimentos ocorreram uma vez por semana, sendo 55 minutos de sessão, totalizando 8 sessões de intervenção. Os materiais utilizados foram: bastões, steps, halteres, caneleiras, escada progressiva, rampa, barras paralelas, garrafas, elásticos, cones, dentre outros objetos, os exercícios foram distribuídos para membros superiores, tronco e membros inferiores, estão descritos na tabela 1. 
Tabela I. Descrição dos exercícios executados nas estações

\begin{tabular}{|c|c|}
\hline Estação 1 & $\begin{array}{l}\text { Exercício ativo de prono-supinação de cotovelo, utilizando garrafinhas, } \\
\text { tubo de pasta. }\end{array}$ \\
\hline Estação 2 & Exercício ativo de marcha frontal e lateral numa escada funcional. \\
\hline Estação 3 & Exercício ativo de inspiração e expiração soprando um catavento. \\
\hline Estação 4 & $\begin{array}{l}\text { Exercício ativo de flexão de ombro segurando uma bola terapêutica com } \\
\text { objetivo de arremessar a bola até o cesto que estará disposto numa } \\
\text { distancia de um metro e meio. }\end{array}$ \\
\hline Estação 5 & $\begin{array}{l}\text { Exercício resistido de flexão de cotovelo a } 90 \text { o com halter de } 1,0 \mathrm{~kg} \\
\text { associado a uma marcha em zigue zague ultrapassando oito cones. }\end{array}$ \\
\hline Estação 6 & $\begin{array}{l}\text { Exercício ativo de subir e descer em dois steps no chão com a distância } \\
\text { de } 20 \mathrm{~cm} \text { entre eles associado ao movimento de agachamento em } \\
\text { bípede. }\end{array}$ \\
\hline Estação 7 & $\begin{array}{l}\text { Exercício ativo de flexão de ombro e rotação de tronco utilizando um } \\
\text { bastão. }\end{array}$ \\
\hline Estação 8 & $\begin{array}{l}\text { Exercício ativo de flexo-extensão de cotovelo e ombro, segurando um } \\
\text { bastão de } 1,0 \mathrm{~kg} \text {. }\end{array}$ \\
\hline Estação 9 & $\begin{array}{l}\text { Exercício ativo de marcha lateral associado ao movimento de rotação } \\
\text { interna seguida de rotação externa mantendo uma flexão de cotovelo } \\
\text { aproximadamente a } 90 \text { o segurando uma vassoura, de modo a simular } \\
\text { atividade cotidiana. }\end{array}$ \\
\hline Estação 10 & $\begin{array}{l}\text { Paciente em pé, com flexão de quadril a } 30^{\circ} \text { aproximadamente e joelho } \\
\text { estendido chutando um objeto a frente. }\end{array}$ \\
\hline Estação 11 & $\begin{array}{l}\text { Exercício ativo de flexo-extensão de cotovelo e ombro seguido de } \\
\text { agachamento em bípede e rotação de tronco, a fim de transferir peças } \\
\text { de madeira de uma cadeira para uma prateleira. }\end{array}$ \\
\hline Estação 12 & $\begin{array}{l}\text { Exercício ativo de flexão de ombro a } 90^{\circ} \text { e flexo-extensão de cotovelo } \\
\text { com as mãos entrelaçadas atingindo um alvo. }\end{array}$ \\
\hline Estação 13 & $\begin{array}{l}\text { Exercício resistido de marcha lateral e frontal sobre o step na barra } \\
\text { paralela utilizando caneleira de } 1,0 \mathrm{~kg} \text {. }\end{array}$ \\
\hline Estação 14 & $\begin{array}{l}\text { Exercício resistido de subir e descer escada e rampa com caneleira de } \\
1,0 \mathrm{~kg} \text {. }\end{array}$ \\
\hline
\end{tabular}

Para a análise estatística, foi utilizado o software Statistical Software for Social Sciences (SPSS Inc. Chicago, IL) versão 18.0, sendo apresentada a estatística descritiva na forma de médias e desvios-padrões. Para verificar a normalidade dos dados, foi utilizado o teste de ShapiroWilk e para comparar os dados de cada paciente, e os valores obtidos nas avaliações funcionais foi utilizado o teste T-student para amostras pareadas, considerando significante o valor de $p \leq 0,05$, para verificar o efeito clínico entre as avaliações foi calculado o Effect Size (ES), pela fórmula de Cohen (d) e a correlação de Pearson para correlacionar o CRE com o TUG.

\section{RESULTADOS}

Dos 15 indivíduos de ambos os sexos inicialmente selecionados para a realização do estudo, apenas 8 foram incluídas no estudo, todas do sexo feminino. Um resumo das características dos participantes está apresentado na tabela I. 
Tabela I. Características dos participantes submetidos a FGCT

Características Participantes $(\mathbf{n}=8)$

\begin{tabular}{lc}
\hline Idade M (anos), DP & $58,62 \pm 9,07$ \\
\hline Local da lesão (Direito \%) & $50 \%$ \\
\hline Tempo diagnóstico M (meses) & $82,5 \pm 58,94$ \\
\hline
\end{tabular}

$\mathrm{N}$ : número de participantes; M: média; DP: desvio padrão.

A análise estatística não revelou diferença significativa entre AV1 $(17,23 \pm 6,84)$ e $\operatorname{AV} 2(12,65 \pm 4,65)$ quanto a mobilidade funcional $(p \leq 0,05)$, apesar disso, o ES mostrou um grande efeito clínico entre as AV1 e AV2 $(0,94)$. A média do CREp foi 3,0 $\pm 0,39$ mostrando um efeito positivo de enfrentamento frente a doença. A correlação de Pearson demonstrou uma correlação moderada, mas não significante entre o questionário CREb com o TUG $(r=-0,618$ e $p=0,102)$.

\section{DISCUSSÃO}

Os participantes deste estudo utilizaram, de alguma forma, o CRE como estratégia de enfrentamento da doença. Apesar da correlação não ter sido significante, esse estudo permitiu a redescoberta da importância da espiritualidade influenciando a clínica e o curso da doença, em termos de apoio e possível variável de prognóstico potencial, uma vez que, os participantes consideram a espiritualidade, em especial, como forma auxiliar de enfrentamento da doença.

Foi observado que após a intervenção em FGCT, houve uma redução do tempo gasto durante a execução da tarefa solicitada no TUG, demonstrando melhora da mobilidade dos participantes, somando-se a um grande efeito clínico para o effect size após 8 sessões.

Embora os alcances religiosos e espirituais sejam condições pouco exploradas na área da saúde, estudos despontam por tal temática, todavia, no que é de conhecimento dos autores, trabalhos com agrupamentos em hemiparéticos em centros de reabilitação ainda são escassos ${ }^{22,23}$.

$\mathrm{O}$ atual estudo soma-se aos poucos que propõem a debaterem o assunto de forma mais pragmática, além de levar à luz a espiritualidade/religiosidade na semântica da intervenção clínica, uma prática que ainda precisa ser dismistificada ${ }^{22}$.

Os pacientes deste estudo, apresentaram um efeito positivo frente a doença através da avaliação do CRE, o que torna esta ferramenta de grande utilidade na prática clínica, uma vez que, com o resultado, a fisioterapia poderá ser capaz de entender a dicotomia entre paciente-terapia frente ao tratamento de saúde com a espiritualidade/religiosidade que é uma condição intrínseca do indivíduo ${ }^{24}$.

Outro aspecto notado é que a população analisada apresenta cronicidade da patologia, então, talvez tenha influenciado no resultado encontrado, pois é observado que quanto maior o tempo de lesão, maior é o enfrentamento negativo da religiosidade/espiritualidade e isso, também, influi diretamente na taxa de adesão ao tratamento ${ }^{22,25}$.

Por outro lado, embora a mobilidade funcional não tenha tido apresentado significância, é visível que a FGCT foi capaz de manter os níveis funcionais dos pacientes, o que remonta para a importância da continuidade destes pacientes neste modelo terapêutico, assim como já relatado na literatura ${ }^{14}$.

As limitações do estudo referem-se ao pequeno número da amostra, variação do tempo de lesão, comprometimento motor heterogêneo e diferentes idades e que somados puderam influenciar nos resultados apresentados e consequentemente na performance motora. Como sugestão para estudos futuros, tais apontamentos apresentados deverão ser considerados para obtenção de resultados mais robustos. 


\section{CONCLUSÃO}

Nota-se que a FGCT não promoveu melhora na mobilidade funcional e nem uma correlação significante entre CRE e TUG ao final de 8 semanas, todavia, foi evidenciado efeito positivo ao enfrentamento da doença e um grande efeito clínico entre as avaliações na mobilidade funcional.

\section{DECLARAÇÃO DE CONFLITO DE INTERESSE}

Os autores declaram não haver qualquer potencial conflito de interesse que possa interferir na imparcialidade deste trabalho cientifico.

\section{REFERÊNCIAS}

1. Segre M, Ferraz FC. O conceito de saúde. Rev. Saúde Pública. 1997, vol.31, n.5, pp.538-542. DOI: http://dx.doi.org/10.1590/S0034-89101997000600016

2. Shanshan L, Stampfer MD, Williams DR, VanderWeel TJ. RELIGIOUS SERVICE ATTENDANCE AND MORTALITY AMONG WOMEN. JAMA Intern Med. 2016 June 01; 176(6): 777-785. DOI: 10.1001/jamainternmed.2016.1615. https://doi.org/10.1001/jamainternmed.2016.1615

3. Saad, M. Masiero, D. Battistella. Espiritualidade baseada em evidências. Acta Fisiátrica 8(3): 107-112, 2001. DOI: https://doi.org/10.5935/0104-7795.20010003. https://doi.org/10.5935/0104$\underline{7795.20010003}$

4. Mesquita, AC. Chaves, ECL. Avelino, CCV. Nogueira, DA. Panzini, RG. Carvalho, EC. A utilização do enfrentamento religioso/espiritual por pacientes com câncer em tratamento quimioterapêutico. Rev. Latino-Am. Enfermagem mar.-abr. 2013;21(2).

5. Caldeira S, Carvalho EC, Vieira M. Entre o bem-estar espiritual e a angustia espiritual: possíveis fatores relacionados a idosos com cancro. Rev. Latino-Am. Enfermagem jan.-fev. 2014;22(1). DOI: 10.1590/0104-1169.3073.2382. https://doi.org/10.1590/0104-1169.3073.2382

6. Ghaffari F, Shamsalinia A, Navabi N, Chafjiri RT. The relationship between the spiritual attitude of the family caregivers of older patients with stroke and their burden. Clin Interv Aging. 2017; 12: 453-458. DOI: 10.2147/CIA.S121285. https://doi.org/10.2147/CIA.S121285

7. VanderWeele, TJ.Tracy, AB, Howard, KK. Health and Spirituality. JAMA. 2017;318(6):519520. DOI:10.1001/jama.2017.8136. https://doi.org/10.1001/jama.2017.8136

8. VanderWeele, TJ. Li, S. Tsai, AC. Kawachi, L. Association between religious service attendance and lower suicide rates among US women. JAMA. Phychiatry. 2016;73(8):845-51. DOI: 10.1001/jamapsychiatry.2016.1243. https://doi.org/10.1001/jamapsychiatry.2016.1243

9. Sousa, FFPR. Freitas, SMFM. Farias, AGS. Cunha, MCSO. Araújo, MFM. Veras, VS. Enfrentamento religioso/espiritual em pessoas com câncer em quimioterapia: revisão integrativa da literatura. SMAD, Rev. Eletrônica Saúde Mental Álcool Drog. Jan.-Mar. 2017;13(1):45-51. DOI: 10.11606/issn.1806-6976.v13i1p45-51. https://doi.org/10.11606/issn.1806-6976.v13i1p45-51

10. Pereira AS, Mathias MB, Freitas DB, Broek VNVD, Barbatto LM, Carvalho AC. Estudo clínico para avaliar o tempo gasto com exercicios físicos durante a fisioterapia de grupo em circuito de treinamento em hemiparéticos crônicos. Colloq Vitae 2016 set-dez; 8(3):29-33. DOI: 10.5747/cv.2016.v08.n3.v173. https://doi.org/10.5747/cv.2016.v08.n3.v173 
11. Billinger S, Arena R, Bernhardt J, Eng J, Franklin B, Johnson C, et al. Physical activity and exercise recommendations for stroke survivors: a statement for healthcare professionals from the American Heart Association/American Stroke Association. Stroke. 2014;45(8):2532-53. DOI: 10.1161/STR.0000000000000022. https://doi.org/10.1161/STR.0000000000000022

12. French B, Thomas L, Leathley M, Sutton C, McAdam J, Forster A, et al. Does repetitive task training improve functional activity after stroke? A Cochrane systematic review and meta-analysis. J Rehabil Med. 2010;42(1):9-14. DOI: 10.2340/16501977-0473. https://doi.org/10.2340/16501977-0473

13. Jeon B, Kim W, Park E. Effect of task-oriented training for people with stroke: a metaanalysis focused on repetitive or circuit training. Top Stroke Rehabil. 2015;22(1):34-43. DOI: 10.1179/1074935714Z.0000000035. https://doi.org/10.1179/1074935714Z.0000000035

14. Carvalho AC, Pereira AS, Oliveira MCS, Franco MR. Fisioterapia em grupo no formato de circuito de treinamento pós-acidente vascular cerebral. In: Associação Brasileira de Fisioterapia Neurofuncional; Garcia CSNB, Facchinetti LD, organizadoras. PROFISIO Programa de Atualização em Fisioterapia Neurofuncional: Ciclo 5. Porto Alegre: Artmed Panamericana; 2018. p. 41-75. (Sistema de Educação Continuada a Distância, v.3).

15. van de Port IGL, Wevers L, Roelse $H$, van Kats L, Lindeman E, Kwakkel G. Cost-effectiveness of a structured progressive task-oriented circuit class training programme to enhance walking competency after stroke: the protocol of the FIT-Stroke trial. BMC Neurol. 2009;9:43. DOI: 10.1186/1471-2377-9-43. https://doi.org/10.1186/1471-2377-9-43

16. Billinger S, Arena R, Bernhardt J, Eng J, Franklin B, Johnson C, et al. Physical activity and exercise recommendations for stroke survivors: a statement for healthcare professionals from the American Heart Association/American Stroke Association. Stroke. 2014;45(8):2532-53. DOI: 10.1161/STR.0000000000000022. https://doi.org/10.1161/STR.0000000000000022

17. Sacco R, Kasner S, Broderick J, Caplan L, Connors J, Culebras A, et al. An updated definition of stroke for the 21st century: A statement for healthcare professionals from the American heart association/American stroke associationAn updated definition of stroke for the 21st century: a statement for healthcare professionals fr. Stroke. 2013;44(7):2064-89. DOI: 10.1161/STR.0b013e318296aeca. https://doi.org/10.1161/STR.0b013e318296aeca

18. Gregson JM, Leathley M, Moore AP, Sharma AK, Smith TL, Watkins CL. Reliability of the Tone Assessment Scale and the modified Ashworth scale as clinical tools for assessing poststroke spasticity. Arch Phys Med Rehabil. 1999; 80(9):1013-16. https://doi.org/10.1016/S00039993(99)90053-9

19. Bertolucci PHF, Brucki SMD, Campacci SR, Juliano Y. The Mini-Mental-State-Examination in an Outpatient Population - Influence of Literacy. Arq Neuro-Psiquiat. 1994; 52(1): 1-7. https://doi.org/10.1590/S0004-282X1994000100001

20. Borges ML. A utilização do coping religioso/espiritual por mulheres submetidas ao tratamento do câncer de mama. Ribeirão Preto, 2015 SP. 
DOI: 10.11606/D.22.2015.tde-04092015-161621. https://doi.org/10.11606/D.22.2015.tde$\underline{04092015-161621}$

21. Cabral ALL. Tradução e validação do teste Timed Up and Go e sua correlação com diferentes alturas da cadeira. 2011. 102 f. Dissertação (Mestrado em Ciências da Saúde) Universidade Católica de Brasília, Brasília, 2011.

22. Johnstone B, Franklin KL, Yoon DP, Burris J. Relationships Among Religiousness, Spirituality, and Health for Individuals with Stroke. Cheryl Shigaki. J Clin Psychol Med Settings, 2008; 15:308313. DOI 10.1007/s10880-008-9128-5. https://doi.org/10.1007/s10880-008-9128-5

23. Giaquinto S, Sarno S, Dall'Armi V, Spiridigliozzi C. Religious and Spiritual Beliefs in Stroke Rehabilitation. Clinical and Experimental Hypertension, 2010 32(6): 329-334. DOI: 10.3109/10641960903443566. https://doi.org/10.3109/10641960903443566

24. Vitorino LM, Low G, Vianna LAC. Assessing the BRIEF spiritual/religious coping scale among older Brazilians, Journal of Religion, Spirituality \& Aging, 2018. ISSN: 1552-8030 (Print) 1552-8049 (Online). DOI: $10.1080 / 15528030.2018 .1474835$.

https://doi.org/10.1080/15528030.2018.1474835

25. Gobatto CA, Araujo TCCF. Coping religioso-espiritual: reflexões e perspectivas para a atuação do psicólogo em oncologia. Rev. SBPH v.13 n.1, Rio de Janeiro, 2010. 